\title{
Design of RANTES-derived Peptides With Enhanced HIV-inhibitory Activity and Derivation of Resistant HIV-I Strains Luca Vangelista* ${ }^{*}$, Renato Longhi ${ }^{2}$, Monica Tolazzi ${ }^{1}$, Vincenzo Pavone ${ }^{3}$ and Paolo Lusso ${ }^{1}$
}

\author{
Address: ${ }^{1}$ Unit of Human Virology, San Raffaele Scientific Institute, Milan, Italy, ${ }^{2}$ CNR-IBRM, Milan, Italy and ${ }^{3}$ Department of Chemistry, \\ University "Federico II", Naples, Italy \\ Email: Luca Vangelista* - vangelista.luce@hsr.it \\ * Corresponding author ‡Presenting author
}

from 2005 International Meeting of The Institute of Human Virology

Baltimore, USA, 29 August - 2 September 2005

Published: 8 December 2005

Retrovirology 2005, 2(SuppI I):PII3 doi:I0.I I86/I742-4690-2-SI-PII 3

We previously identified the major structural determinants of CCR5 binding and HIV blockade in RANTES, describing linear RANTES-derived peptides with biological activity in the low micromolar range (Nardese et al., 2001). To deepen our understanding of RANTES structure-function relations and obtain more potent antiviral peptido-mimetics, we have extensively mutagenized the prototypic peptide, R11-29. This presents two clusters of hydrophobic residues at its termini, (corresponding to RANTES N-loop and b1-strand) connected by a positivelycharged linker. Single or multiple alanine substitutions within the N- or C-terminal hydrophobic clusters resulted in a dramatic loss of antiviral activity, whereas deletion of selected residues within the hydrophilic linker had no major functional consequences. Based on RANTES 3D structure, we designed a series of modified peptides, resulting in a progressive increase in specific antiviral activity. These peptides also displayed anti-inflammatory properties blocking RANTES-elicited lymphocyte chemotaxis. Through serial passages in culture in the presence of increasing concentrations of the most effective antiviral peptides, we have derived variants of the R5 HIV-1 isolate $\mathrm{BaL}$ resistant to the peptide inhibitory activity. Complete sequencing of the envelope genes from such variants is currently underway. Our results provide new insights into the structure of the receptor-binding region of RANTES and identify new antiviral peptides that may be instrumental in the development of effective HIV-1 entry inhibitors. 